\title{
AS REALIDADES DE UMA AVENTURA DOCUMENTÁRIA
}

\section{LAS REALIDADES DE UNA AVENTURA DOCUMENTAL}

\author{
Stephanie Manfroid - stephanie.manfroid@mundaneum.be \\ Diretora dos Arquivos - Instituto Mundaneum, Bélgica.
}

\begin{abstract}
RESUMO
Introdução: Sob o vocábulo Mundaneum se escondem instituições variadas. Hoje, esse é o nome de um centro de arquivos localizado na Bélgica e que conserva, preserva e valoriza o patrimônio sobre a bibliografia, a documentação e os registros imagéticos. Essa herança remonta ao século XIX, quando o Instituto Internacional de Bibliografia decidiu pela criação do Repertório Bibliográfico Universal.

Objetivo: A proposta deste trabalho é apresentar e descrever esse vasto repertório sintético, uma realização original em sua forma e em seu funcionamento. A denominação atual de "Google de Papel" serve bem à essa iniciativa, que promoveu a Classificação Decimal Universal (CDU). Sendo a primeira etapa da colaboração internacional que tinha por objetivo a paz mundial reuniu bibliógrafos e outros personagens de interesse mais amplo. Essa cooperação sob o espírito internacional é maior, se manifesta em novas esferas como a União das Associações Internacional no seio do Palácio Mundial-Mundandeum. Após a primeira Guerra Mundial, Bruxelas abriga o conjunto dessas iniciativas no seu centro internacional, dotado de um museu, uma biblioteca, do centro de documentação especializada, e um local de debate.

Metodologia: trata-se de um trabalho teórico, com fundamentação histórica e perfil descritivo, que procura problematizar e interpretar a construção histórica dos projetos que envolvem a história do Mundaneum.

Resultados: Reconhece-se aqui que uma universidade internacional é organizada para atender às ambições dos responsáveis por essas instituições. Paul Otlet e Henri La Fontaine, os pais por detrás de tal ideal, engendram essas instituições insuflando a cooperação intelectual internacional como um salto particular. De fato, eles lançam as bases da padronização das bibliotecas e estendem seu campo de aplicação às associações privadas.

Conclusões: Graças a esse diálogo permanente e à cooperação em grande escala, o universalismo do conhecimento alcança um grau de realização inédito, sem, para tanto, impor a paz.
\end{abstract}

Palavras-chave: Instituto Internacional de Bibliografia. Mundaneum. Repertório Bibliográfico Universal. 


\section{INTRODUCTION}

Le Mundaneum est un centre d'archives privées installé à Mons qui gère un patrimoine exceptionnel pour l'histoire de la Bibliographie. Son équipe composée de 15 personnes occupe une infrastructure originale de type Art déco dans une localité d'inspiration médiévale. Rénové à deux reprises, en 1997 pour accueillir le public et en 2014 pour offrir les conditions de conservation recommandées par le Conseil International des Archives, il réunit une salle d'expositions temporaires, un dépôt d'archives, une salle de lecture et une salle pédagogique pour les animations. Les expositions sont mises sur pied pour valoriser l'histoire de l'institution, les valeurs de la démocratie et susciter le débat autour de la paix. Le centre d'archives est quant à lui spécialisé dans des thèmes particuliers en raison des collections qu'il conserve : le féminisme, le pacifisme et l'anarchisme. Son patrimoine contribue à bien des égards à l'histoire culturelle européenne mais cette dimension s'élargit à un territoire plus vaste et est également internationale.

\section{PROCÉDURES CONTEXTUALISATION} MÉTHODOLOGIQUE ET

Pour comprendre l'institution et son patrimoine, il est important de retracer l'histoire de son développement, évoquer les hommes qui l'ont enrichie et les collections élaborées. Ceci est un travail théorique avec la fondation historique et un profil descriptif, qui vise à discuter et interpréter la construction historique de projets impliquant l'histoire du Mundaneum.

C'est au service de la connaissance que des institutions et des hommes ont coopéré pour créer un centre international à Bruxelles après la première guerre mondiale. Imaginé sous des formes diverses et dans des lieux particuliers de la capitale belge, ce centre intellectuel émerge en réalité à la fin du XIXe siècle alors que l'Etat belge est une nation florissante aux réussites économiques et industrielles qui la 
propulse parmi les plus prospères d'Europe. Les conséquences sociales et humaines de cette prospérité sont désastreuses pour la population ouvrière belge. Dès lors la dimension sociale de cette réussite agite rapidement le milieu intellectuel, politique et industriel. La sociologie et les sciences sociales trouvent dans ce contexte un terrain favorable à une réflexion. L'université de Bruxelles est d'ailleurs l'endroit où le débat aboutit à la création d'une université dissidente, l'université nouvelle mais aussi à la création d'un institut dédié à la sociologie grâce au soutien de l'industriel et philanthrope Ernest Solvay. Notons aussi qu'une crise politique agite le pays sur fonds de l'extension du suffrage. Ces paramètres favorisent l'émergence d'une génération d'hommes progressistes qui se retrouvent notamment au sein du Parti Ouvrier Belge (parti socialiste). Toute cette agitation pourrait sembler éloignée de la création d'un institut spécialisé, l'Institut international de Bibliographie. Mais il n'en est rien. L'Institut entretient une relation étroite avec ces deux considérations. La première section bibliographique est créée vers 1890 en Belgique au sein de la Société d'études sociales et politiques sous l'impulsion d'Auguste Couvreur. Cette société innove réellement avec la création de ce service bibliographique et sa bibliothèque, chargé de classer et cataloguer tous les documents et tous les faits relatifs aux questions économiques et sociales écrits en n'importe quelle langue, survenus en n'importe quel pays. Le bulletin bibliographique de la revue sociale et politique en publie alors les résultats. Le décès de son fondateur en 1894 modifie le développement de cette société dans laquelle le secrétaire n'est autre qu'Henri La Fontaine. Désormais, c'est l'Office International de Bibliographie sociologique qui édite cette bibliographie des sciences sociales dans le cadre de l'Institut des Sciences sociales, initié en 1894 par le même Ernest Solvay. Ce dernier est convaincu de l'intérêt des sciences pour résoudre les maux de la société. II saisit l'opportunité de créer des instituts qui étudient méthodiquement les questions économiques et sociales. L'institut s'adjoint lui aussi un service bibliographique sous 
l'impulsion des deux directeurs, Paul Otlet et Henri La Fontaine. Pour eux, il s'agit d'élargir leurs activités sur une base plus large et ambitieuse: droit, statistique, économie politique, économie sociale. Cet élargissement se justifiait par l'accélération de l'édition des livres dans le domaine particulier des sciences sociales et l'obligation de soutenir l'accès aisé à ces ressources. Quatre services se mettent alors en place: le service des publications bibliographiques, le service des répertoires bibliographiques, le service des recueils de faits sociaux et la bibliothèque. Le répertoire bibliographique est composé de fiches mobiles regroupées par thème. L'OIBS achève alors son parcours bibliographique quand est créé l'Institut International de Bibliographie en 1895 qui poursuit l'ambition de créer des offices nationaux dans les pays étrangers et un répertoire ouvert à toutes les disciplines. L'imminence d'une conférence prévue en 1896 par la Royal Society de Londres invite le projet belge à se développer plus vite. Unifier les méthodes bibliographiques, adopter une classification bibliographique universelle, élaborer un répertoire bibliographique embrassant tous les domaines de la connaissance et mettre en place la coopération internationale sont les nouvelles missions de cet Institut International de Bibliographie. La convocation de la Conférence bibliographique de septembre 1895 avalise ce projet. La Classification décimale universelle est reconnue dans la foulée comme classification internationale. En réalité, deux entités voient le jour, I'OIB et I'IIB. La première se concentre sur le répertoire à travers la rédaction des fiches et sa publication. La seconde constituée d'un réseau de membres se spécialise dans la bibliographie et son outil, la CDU. Ensemble elles publient le bulletin de l'Institut et la Bibliographia Universalis Mundaneum. Tout comme les premiers services de Bibliographie, le siège des activités se situe à l'hôtel Ravenstein, véritable viviers d'association moderne et scientifique. La collaboration internationale facilite cette entreprise qui bénéficie rapidement de bureaux internationaux dans les grandes villes comme Paris ou Zürich. 
Ce répertoire connaît un développement sans précédent entre 1895 et 1930. II renseigne sur les publications de tous les temps, de tous les pays et relatives à toutes les matières, et ce quel que soit le lieu de conservation de ces publications. Son universalité est sans conteste. Quelques millions de fiches le composent (plus de 14 millions). Son format et sa rédaction sont standardisés. La CDU résout la question du classement thématique des fiches. Deux modes de rangement par titre et par nom d'auteur facilitent la consultation des fiches du répertoire. Elles sont ainsi rangées dans des casiers-tiroirs selon l'ordre de succession des nombres des tables systématiques de la CDU. Autre avantage, chaque tiroir peut accueillir de nouvelles fiches qui viennent s'intercaler facilement en raison de leur mobilité. Leur maintien dans chaque tiroir est assuré par une tige métallique et un bloc triangulaire qui donne un confort à la consultation. Autre originalité du répertoire, il est constitué de répertoires complémentaires qui ont trait au courrier reçu, à la gestion du personnel, aux publications ou à l'inventaire des collections. II compose une œuvre originale reconnue depuis 2013 par I'Unesco et son programme mémoire du Monde. Plus de 200 meubles fichiers sont conservés et exposés dans l'espace des expositions temporaires servant à la fois de réflexion sur cette initiative universaliste et de témoignage des efforts des idéalistes de la connaissance.

Le répertoire ainsi centralisé ne constitue qu'une étape d'un travail général de standardisation qui inclut les bibliographies nationales. La publication du manuel de la Classification décimale universelle en 1905 formalise la notation thématique des livres. Les règles pour les notices bibliographiques, les notices catalographiques internationales, les formats de documents et l'emploi d'un type de mobilier précis sont applicables pour tous les professionnels dans leur bibliothèque, par les sociétés savantes nationales ou des associations internationales. En 1911, l'IlB compte pas moins de 790 membres et 22 personnes dans son équipe. Les sections nationales sont au nombre de onze. Les trois sections internationales sont réparties par discipline. La section française 
se structure très rapidement dès 1896. En 1899, elle se transforme en un Bureau Bibliographique de Paris sous l'impulsion notamment de Charles Gariel et du général Hyppolite Sébert. Sous l'influence du militaire à la retraite passionné de photographie et impliqué dans la structuration du mouvement espérantiste, Paul Otlet s'intéresse à la photographie capable de représenter un objet sans passer par une langue. Au sein même du BBF, on retrouve un nombre important de membres répartis entre les scientifiques, les bibliothécaires et les libraires-imprimeurs. Après guerre, les milieux industriels et commerciaux se mélangent aux bibliothécaires et chartistes. Ils arrivent à former malgré leur sphère d'activités différentes une culture autour des problèmes de documentation.

D'autres pays rejoignent l'IIB graduellement. Les pays européens sont fortement représentés avant 1914. Des institutions américaines coopèrent néanmoins en matière de Bibliographie : USA, Uruguay, Mexique, Brésil et Argentine. Tout comme c'était le cas au BBF, les membres sont assez variés : bibliothécaires, scientifiques mais aussi acteurs de la vie économique et industrielle. Généralement, ils utilisent la classification décimale dans la mise en ordre des livres. Enfin, ils produisent des bibliographies spécialisées qui sont publiées dans les Bibliographia Universalis.

Ces rapprochements autour de I'llB amènent de nouvelles réflexions sur les moyens d'organisation de la documentation au niveau international. L'information considérée comme une ressource est conçue pour devenir un système mondial imaginé comme un réseau où l'information circule. À la notion du livre support qui clôt le texte s'est substituée celle de document, où l'information n'est plus limitée à la frontière de la page, ni à celle du pays.

Dès le début du XXe siècle, c'est donc assez naturellement que les travaux de I'llB s'orientent vers d'autres supports de l'information. Les livres ne sont pas les seuls détenteurs de la connaissance : la presse et l'image jouent aussi un rôle important dans l'accès au savoir. 
Le concept de documentation se structure peu à peu. Des collections sont organisées en fonction du type du support et des sections spécialisées sont créées : le répertoire universel de documentation, la Bibliothèque collective des Sociétés savantes, l'institut international de Photographie et le répertoire iconographique universel, le musée international de la presse et l'Atlas Universel ou "Encyclopaedia Universalis Mundaneum ». Ces sections adoptent une méthode commune, la CDU, et forment une nouvelle unité générale dénommée l'Encyclopédie documentaire.

\section{RÉSULTATS HISTORIQUES}

Le projet de bibliothèque universelle constitue l'une des préoccupations majeures de Paul Otlet. En effet, que vaut une notice bibliographique sans une bibliothèque et ses livres ? Indéniablement, la bibliothèque est le complément logique du RBU. Elle réunit autour d'elle près de 40 sociétés scientifiques et leurs bibliothèques respectives. Elaborée sur la base du principe de coopération intellectuelle, cette bibliothèque préserve l'autonomie de chaque société tout en réunissant son catalogue et ses collections en un même lieu. Etant donné le caractère spécialisé de chacune d'elle, elle donne accès à une vaste bibliothèque universelle. La centralisation des services, la réalisation d'inventaires communs et la publication d'un catalogue commun définissent les avantages d'une bibliothèque collective. Elle abrite un service de renseignements sur les organismes belges actifs dans le domaine des sciences, des lettres et des arts qui a fait l'objet en 1908 de la publication d'un annuaire.

Le répertoire universel de documentation se structure sur le constat de l'accélération des publications scientifiques et leur nécessaire accessibilité. Cet outil de collecte et de mise à disposition de l'information comprend des fiches structurées d'informations : auteur, pays, sujet date. La coupure de presse, la brochure, soit la source correspondante est ainsi annexée. Cette nouvelle forme d'encyclopédie 
permet un accroissement constant des informations et une mobilité unique. Chaque information est indépendante et s'intègre très aisément au corpus sans devoir attendre des délais d'impression, ni même les financer. L'utilisation des publications s'effectue une sélection selon leur intérêt et leur sujet. Trois sections distinctes sont ainsi organisées : les dossiers biographiques, les dossiers thématiques et les dossiers géographiques. La diversité des auteurs et l'actualisation des informations se poursuivent de manière automatique.

Un Musée de la Presse est créé en 1906 sous l'influence de Paul Otlet quand il devient responsable d'une association, I'Union de la Presse Périodique. L'étude de la société est effectuée par le recours à ce média. II offre l'avantage de représenter la diversité sociale dans sa dimension locale, régionale et nationale. Des collections de spécimens de périodiques et journaux sont réunis. En 1911, les collections deviennent internationales. Des bibliographies, des annuaires de la Presse, des ouvrages sur l'histoire des journaux et périodiques, des contributions sur l'histoire de la presse, la législation, le droit d'auteur et la liberté d'écrire sont également réunis dans ce Musée international de la presse (MIP). La presse offre le miroir d'une époque riche en spécimens de tous les horizons.

L'iconographie bénéficie, à partir de 1903, d'une attention particulière au sein de l'IIB. Certains membres comme le Général Sébert l'intègre dans le processus d'information avec des particularités propres. Une section à part entière lui est consacrée dans le nouvel Institut international de Photographie. II a pour but de réunir l'ensemble des connaissances en matière de photographies. C'est Ernest de Potter, photographe amateur et collectionneur, qui dynamise ce projet. II offre ainsi sa collection personnelle composée de plaques de verre, de photographies, de cartes postales et d'affiches qui ébauche le Répertoire iconographique universel. Une bibliothèque spécialisée, des dossiers documentaires (constitués d'images exclusivement) et un répertoire bibliographiques des sciences photographiques forment les collections 
de cet IIP. La Revue internationale de photographie devient la publication de cette section à part entière. Des congrès internationaux sont organisés avec des partenaires tels que le Musée Suisse des Photographies Documentaires (Genève) et le Musée des photographies Documentaires (Paris). Les questions débattues sont en rapport avec le rôle de la photographie dans la documentation ou l'histoire, les dépôts d'archives photographiques ou encore la conservation de l'image et ses supports. Lors du Congrès de Marseille de 1906, la notoriété de l'IlP devient internationale. Désormais, il élabore le catalogue universel des collections de photographies documentaires.

L'Atlas Universel ou Encyclopaedia Universalis Mundaneum est élaboré plus tardivement, après la première guerre mondiale. II est largement influencé par la nouvelle Société des Nations qui favorise une approche internationale dans l'éducation. Cette Encyclopédie illustrée, composée de tableaux de grand format sur planches mobiles, synthétisent les sources d'informations variées (fiches bibliographiques, livres, presse, dossiers documentaires). De manière systématique, chaque planche contient des informations à un endroit bien défini : le titre, un texte explicatif, la date, les sources documentaires et le nom du rédacteur de la fiche. La priorité de la composition est donnée au dessin couleur ou noir et blanc, à la photographie, à la carte ou au diagramme. L'Atlas de la préhistoire est le spécimen le plus original car il est composé d'aquarelles artistiques.

Dans cette section, la CDU est utilisée pour le regroupement des planches. Quatre séries générales ou Atlas existent : les sciences et les divers ordres d'activités, l'histoire, la géographie et la vie internationale. Un atlas de la civilisation universelle destiné à l'enseignement a notamment été créé par Paul Otlet et Anne Oderfeld. Dans ce cas, le format de I'Atlas s'adapte à une manipulation aisée. II adopte un format réduit de la taille d'une simple feuille de papier. La commission internationale du matériel didactique du Bureau International d'Education 
a participé à cette édition. Les tableaux sont aussi filmés et constituent sous cette forme l'Encyclopaedia Microphotica.

Plus de 8000 panneaux différents et plusieurs centaines de films composent cette Encyclopédie unique développée au sein du Mundaneum.

Sur dix années d'existence, les organismes créés dans la foulée de l'Institut International de Bibliographie offrent des visages variés et modernes. Un constat s'impose : la connaissance est partout. Les formes en sont diverses. La Classification Décimale Universelle est son langage et outil de travail pour organiser et interroger les collections. Mais une préoccupation demeure. La concorde internationale basée sur un dialogue culturel est à construire. La paix est la prochaine étape de la société idéale. C'est la raison pour laquelle un organisme entièrement voué à la collaboration entre les institutions internationales est fondé par Paul Otlet et Henri La Fontaine. Cette initiative privée, I'Union des Associations Internationales, se démarque de la diplomatie officielle. Elle s'organise et se réunit à Bruxelles lors des Congrès internationaux. En 1910, l'exposition internationale et universelle de Bruxelles lui permet de créer un musée. II affirme ainsi la réalité de l'internationalisme. Mais c'est véritablement après la guerre qu'il parvient à s'imposer aux côtés de la Société des Nations et il y soutient la coopération intellectuelle. Cet organisme sert de laboratoire à des ambitions politiques plus grandes. À sa manière et avec des moyens très modestes, I'UAl s'engage dans la voie de la concorde avec l'organisation de congrès internationaux, d'une université internationale et le projet de Cité mondiale. Tous les instituts bibliographiques et documentaires sont alors associés à l'activité de I'UAI qui bénéficie d'installations au Cinquantenaire.

\section{CONCLUSIONS}

Cette présentation synthétique de la réalité documentaire de l'IlB occulte à la fois les difficultés et les soutiens que rencontrent le partage de la documentation et la diffusion de la connaissance universelle au 
début du XXe siècle. On aurait tort de voir ici la réussite ou l'échec d'institutions documentaires dans le domaine de la politique internationale par exemple. Réduire ces initiatives à cette simple équation ne permet pas de comprendre leur apport et leur originalité. La concurrence du système de classification développé par Dewey et l'hostilité des milieux scientifiques ont constitué des obstacles, sans être les seuls, à la légitimité d'un processus entièrement basé sur un idéal collectif. Les années trente sont une période critique tant pour les défenseurs de la paix que pour la crédibilité de la Société des Nations. Elle déçoit et n'offre pas encore une réponse efficace aux nationalismes qui perturbent les relations internationales. Le soutien de l'Etat belge à cette entreprise disparaît lui aussi peu à peu. L'ouverture des débats politiques internationaux et la promotion d'une cité mondiale sont dans l'impasse. Les associations internationales réunies au sein de l'UAl sont l'objet d'un intérêt particulier de la Deutsche -Kongress Zentrale, située en Allemagne. Au cours de la seconde guerre mondiale, elle prend possession d'une partie des documents de l'UAl pour surveiller ses membres et ses groupes. La disparition des pères fondateurs de l'IlB marque l'arrêt d'une aventure documentaire inédite. Mais qui étaient les promoteurs de ces développements ? Enfin quel matériau est parvenu jusqu'à nous?

Parmi les promoteurs de l'llB et de l'UAI, deux personnalités au service de la connaissance et de la paix émergent, Henri La Fontaine et Paul Otlet. Elles se définissent par une même origine sociale, économique et intellectuelle. Leur esprit encyclopédique, prêt à investir tous les domaines du savoir, est aussi important que leur souci d'appropriation collective et rationnelle des informations. Leur formation en droit leur ouvre tout un univers intellectuel où se confond la vie judiciaire et littéraire du pays. C'est autour du réformisme social qu'ils se rencontrent aux alentours de 1890.

Quand Henri La Fontaine se spécialise dans le droit international, ses études universitaires sont en cours entre 1873 et 1877 . Par la suite, 
il travaille auprès de bureaux d'avocats animés par des personnalités politiques de premier plan. Son implication dans la vie associative belge lui offre des opportunités de rencontres et de collaborations : l'Association pour l'enseignement professionnel des femmes, l'école professionnelle Bischoffsheim, la franc-maçonnerie, l'association libérale, le Parti Ouvrier Belge, le Club Alpin Belge, le cercle des étudiants et anciens étudiants socialistes et la société belge de l'arbitrage et de la paix. Parallèlement à ces activités professionnelles, il s'investit auprès de son mentor Auguste Couvreur dans la société d'études sociales et politiques. II navigue ainsi entre conscience politique progressiste, pacifisme et féminisme.

Au cours des années 90, sa position s'affirme avec des responsabilités plus importantes. Les questions de paix, le droit des femmes et le suffrage universel sont des matières qui le mobilisent tant en Belgique qu'à l'étranger. II devient entre autre Sénateur pour le Parti Ouvrier, organisateur des congrès universels de la Paix, professeur à l'université et fondateur du groupement féministe, la ligue du droit des femmes. II a à son actif de nombreuses publications dont une bibliographie sur l'alpinisme. Sa participation à la Société d'études sociales et politiques l'amène à diriger la section bibliographique. À partir de 1893, il s'associe à Paul Otlet pour la gestion de cette section qui se mue en un Institut International de bibliographie sociologique. Pour les besoins de I'IIB, il traduit en français les tables de la classification décimale de Dewey. Henri La Fontaine participe à la première Conférence de la Paix de La Haye. Son discours s'affirme autour de l'arbitrage et du droit international. En 1907, il dirige le Bureau International de la Paix. Son internationalisme le conduit à la fois dans les associations pacifistes et socialistes. II rejoint le Bureau socialiste international lorsque ses assemblées internationales extraordinaires internationales sont consacrées à la situation internationale. Quand il obtient le Prix Nobel de la Paix, en 1913, l'indifférence de son pays est grande tout comme son absence aux conférences de la paix. Durant la 
première guerre mondiale, il est en exil en Grande-Bretagne puis EtatsUnis où il peaufine sa conception de la société internationale. Sa Magnissima Charta est publiée en 1917 à Boston. Dans cet ouvrage, il détaille les 127 articles de l'organisation du pouvoir judiciaire international. Enfin, il juge nécessaire d'organiser une force de contrainte armée. Lors de la conférence de la Paix de Versailles en 1919, il assiste aux débats en tant que conseiller technique. L'année suivante, il est membre de la première délégation belge envoyée à la Société des Nations. Ses prises de position en faveur notamment du peuple arménien l'évincent des réunions de cette assemblée internationale. Sa carrière politique continue au niveau national jusqu'en 1932. C'est au cœur du centre international intellectuel qu'il fait entendre sa voix pour une organisation du travail intellectuel. II participe encore à la Xe Conférence internationale de Bibliographie en 1931.Mais cette année-là, I'IIB se mue en un Institut international de documentation. C'est avec beaucoup de regrets qu'il assiste à cette évolution. Henri La Fontaine est ainsi un représentant incontournable du pacifisme belge.

Le parcours de Paul Otlet se distingue singulièrement de son partenaire que nous venons d'évoquer. II incarne plus volontiers le volet des connaissances au service de la paix. L'entreprise familiale et la figure du père vont largement dominer sa personnalité et son entrée dans la vie professionnelle. Edouard Otlet fonde sa fortune sur sa société de construction de tramways. II en établit partout dans le monde et plus particulièrement en Europe. Le terme entreprendre définit parfaitement l'état d'esprit des Otlet. D'autre part, cet univers familial, très étendu, est actif dans toutes les dimensions de la société. L'art, la botanique, le commerce, l'économie, le tourisme se confondent avec un ses membres. Pour illustrer cette particularité, notons que Paul Otlet voyage avec son père en Russie alors qu'il est âgé d'à peine 17 ans. Succéder à son père à la tête de cette société est une évidence. Mais, il ne s'agit pas de son ambition personnelle. Lui, qui a déjà publié à 14 ans, s'accomplit dans l'étude et les livres. II poursuit des études de droit 
et effectue son stage en 1890 chez un proche de la famille, Edmond Picard. Avec lui, il travaille à une encyclopédie du droit belge et plus particulièrement sa jurisprudence. C'est durant cette expérience qu'il découvre et pratique la bibliographie. C'est le travail mené par la société d'études sociales et politiques qui le conduit à créer une structure bibliographique à part entière. En 1895, il partage son temps et son énergie entre cette passion et l'entreprise de son père. II organise ainsi sur des terrains de chasse, situés sur la côte belge (Westende), une station balnéaire d'un genre nouveau où l'architecture est privilégiée. II en imagine les moindres détails de l'électrification à l'esthétisme. L'organisation de cette cité laisse des traces chez cet homme avide de connaissances et de coopération. À partir de l'Union des Associations Internationales, il concentre dès 1910 toute son énergie à la conception d'une Cité Mondiale, appelée à devenir la capitale intellectuelle et morale de l'humanité. II sollicite des personnalités diverses autour de ce projet : Hendrik Andersen ou Le Corbusier. Mais cette expérience tourne court.

Entre-temps, l'homme est devenu incontournable en Belgique dans le domaine du livre. Depuis 1906, il est à la tête du musée du Livre. On le sollicite pour la création du premier Ministère de la Culture en Belgique (des Arts et des Sciences). Au décès de son père en 1907, sa disponibilité pour les instituts, qui ont vu le jour dans le sillage de l'IIB, augmente. II s'investit aussi dans l'Union des périodiques belges et en devient le président.

La première guerre mondiale affecte particulièrement Paul Otlet. Son fils, Jean, décède sur les champs de bataille. II résulte de cette disparition un engagement plus prononcé dans le pacifisme. Dès octobre 1914, il publie La fin de la guerre. Traité de Paix général. Deux ans plus tard, il écrit les problèmes internationaux et la guerre à Genève.

Après la guerre, sa confiance en la Société des Nations est telle qu'il se positionne en faveur de la création de la commission internationale de coopération intellectuelle $(\mathrm{ClCl})$. II collabore aussi 
activement avec le Bureau international d'Education sur l'Encyclopedia Universalis Mundaneum.

Les années trente sont difficiles pour les institutions et les idées qu'il défend. Le Mundaneum est fermé en 1934 sur ordre du gouvernement. Par ailleurs, il publie son ouvrage majeur, Le Traité de documentation. Le livre sur le livre. dans lequel il définit tous les supports de la connaissance. Sa vision va au-delà du livre conventionnel et il esquisse une présentation moderne des outils de consultation. Le livre téléphoté en est l'exemple le plus parlant. Quelques schémas sont intégrés dans l'ouvrage. Mais, l'encyclopédie qu'il rédige (EUM) en contient davantage, révélant ce nouveau mode d'expression philosophique. Enfin, son ouvrage Monde, essai d'Universalisme publié en 1935, constitue un réel apport sociologique à ses réflexions. II s'engage même à définir ce que nous reconnaissons dans le web 2.0. La Mondothèque fait partie des nouveaux mobiliers qu'il décrit. Elle contient les outils complémentaires de la connaissance et ses supports comme des fenêtres sur nos ordinateurs ou le mutlimédia.

L'apport de Paul Otlet en matière d'organisation de la connaissance est majeur à la fois d'un point de vue théorique mais également pratique. Les collections élaborées en collaboration avec son partenaire Henri La Fontaine sont là pour le prouver. Elles ont connu certes des difficultés avec la fermeture en 1934, l'occupation allemande et ses diverses conséquences. La première est le déménagement vers d'autres lieux exigé par les allemands en 1941. La seconde est la mainmise sur des archives par la Deutsche -Kongress Zentrale l'année suivante. Transférées à Berlin, ces archives y resteront jusqu'à la fin de la guerre. Une mission européenne dirigée par les américains, et notamment l'Institut Hoover, découvre cette unité originale. Depuis lors, elle est conservée à Stanford.

Le Mundaneum actuel conserve une bonne partie des archives des fondateurs et des collections qu'ils ont créées entre 1895 et 1944. Les successeurs des deux hommes ont continué d'enrichir les 
collections dans l'association les Amis du Palais Mondial. En Belgique, c'est donc pas moins de 6000 mètres courant d'archives qui sont conservées. Elles comprennent toutes les unités que nous avons exposées, des répertoires bibliographiques, documentaires et iconographiques jusqu'aux plaques de verre, les photographies ou affiches. Parmi cet ensemble, on retrouve quelques pièces originales comme le Prix Nobel d'Henri La Fontaine attribué en 1913, le film témoignant de la visite d'Andrew Carnegie (1913) au Musée international et un film sonore d'une visite guidée de Paul Otlet au Palais Mondial en 1932. S'il est évident que de nombreux documents ont disparu depuis le décès des fondateurs, le centre d'archives a pour mission de préserver ce qui existe, tout en proposant des inventaires accessibles. Enfin, la valorisation de ce patrimoine s'opère de diverses manières tant par les expositions que les publications. D'autre part, les conférences et les colloques que nous organisons ou auxquels nous assistons mettent en évidence ce patrimoine et cette histoire originale. De nombreux pans restent à découvrir.

Je désire enfin adresser mes remerciements aux organisateurs de ce colloque de I'UNIRIO et I'UFRJ dans leurs titres respectifs et qualités: Gustavo Saldanha, Andre Vieira de Freitas Araujo, Giulia Crippa, Lena Vânia Ribeiro Pinheiro. Enfin, ma gratitude s'adresse également à Georgete Medleg Rodrigues de l'UNB et à Carlos Henrique Juvêncio.

\section{BIBLIOGRAPHIE}

CENT ans de l'Office International de Bibliographie, 1895-1995: les prémisses du Mundaneum, Mons, éditions du Mundaneum, 1995.

CORNILLE, Raphaèle; MANFROID, Stéphanie; Valentino, Manuela. Le Mundaneum: les archives de la connaissance. Bruxelles: Le Mundaneum, 2008. 
CROMBOIS, Jean-François. L'univers de la Sociologie en Belgique de 1900 à 1940. Bruxelles: Editions de l'Université de Bruxelles, 1994.

DUBOIS, Sophie. L'Hôtel ravenstein: un enjeu pour les Sociétés Savantes. Bruxelles: Université Libre de Bruxelles, Faculté de philosophie et Lettres, 2001-2002. Mémoire Inédit.

FAYET-SCRIBE, Sylvie. Histoire de la documentation en France: culture, science et technologie de l'information 1895-1937. Paris: CNRS, 2000 .

HENRI, La Fontaine. Prix nobel de la paix en 1913: un belge épris de justice. Bruxelles: Editions Racine, 2012.

LABORATOIRES et réseaux de diffusion des idées en Belgique (XIXeXXe siècles). Bruxelles: Editions de l'Université de Bruxelles, 1994.

LEVIE, Françoise. L'homme qui voulait classer le monde: Paul Otlet et le Mundaneum. Bruxelles: Les impressions nouvelles, 2006.

OTLET, Paul. Fondateur du Mundaneum (1868-1944). Architecte du savoir, artisan de paix. Bruxelles: Les impressions nouvelles, 2010.

RENOLIET, Jean-Jacques. L'UNESCO oubliée: la société des nations et la coopération intellectuelle (1919-1946). Paris: Publication de la Sorbonne, 1999.

\section{Title}

The realities of a documentary adventure

\section{Abstract}

Introduction: Under the Mundaneum word are hidden various institutions. Today, this is the name of a center of archives situated in Belgium and that conserves, preserves and values the equity of the bibliography, documentation and imagetic records. This heritage dates back to nineteenth century, when the International Institute of Bibliography decided to create the Universal Bibliographic Repertory.

Objective: The purpose of this paper is to present and describe this vast synthetic repertoire, a unique achievement in its form and in its operation. The current name of "Paper Google" serves well to describe this initiative, which promoted the Universal Decimal Classification (UDC). As the first step of international collaboration that was to world peace met bibliographers and other characters of wider interest. This cooperation under international spirit is greater, manifests itself in new areas such as the International Associations Union within the World Palace-Munandeum. After the first World War, Brussels 
is home to all of these initiatives in its international center, which boasts a museum, a library, the center of relevant literature, and a place of debate.

Methodology: it is a theoretical work with historical foundation and descriptive profile, which aims to discuss and interpret the historical construction of projects involving the history of Mundaneum.

Results: It is recognized here that an international university is organized to meet the ambitions of running these institutions. Paul Otlet and Henri La Fontainethe parents behind such an ideal, engender these institutions inflating the international intellectual cooperation as a particular jump. In fact, they are laying the foundations of standardization of libraries and extend its scope to private associations.

Conclusions: Thanks to this continuous dialogue and cooperation on a large scale, the universalism of knowledge reaches a degree of unprecedented achievement, without, therefore, impose peace.

Keywords: International Institute of Bibliography. Mundaneum. Universal Bibliographic Repertory.

\section{Titulo}

Las realidades de una aventura documental

\section{Resumen}

Introducción: Bajo la palabra Mundaneum esconden diversas instituciones. Hoy en día, este es el nombre de un centro de archivos que se encuentran en Bélgica y que conserva, preserva y valora el patrimonio neto de la bibliografía, documentación y registros pictóricos. Este patrimonio se remonta al siglo XIX, cuando el Instituto Internacional de Bibliografía decidió crear el Repertorio Bibliográfico Universal.

Objetivo: El propósito de este trabajo es presentar y describir este vasto repertorio sintético, un logro único en su forma y en su funcionamiento. El nombre actual de "Papel Google" sirve bien a esta iniciativa, que promueve la Clasificación Decimal Universal (CDU). Como primer paso de la colaboración internacional que fue a la paz mundial se reunió bibliógrafos y otros caracteres de interés general. Esta cooperación bajo el espíritu internacional es mayor, se manifiesta en nuevas áreas, como la Unión Internacional de Asociaciones en el Palacio Mundial-Munandeum. Después de la primera Guerra Mundial, Bruselas es el hogar de todas estas iniciativas en su centro internacional, que cuenta con un museo, una biblioteca, el centro de la literatura relevante, y un lugar de debate.

Metodología: se trata de un trabajo teórico con fundamento histórico y perfil descriptivo, cuyo objetivo es analizar e interpretar la construcción histórica de los proyectos relacionados con la historia de Mundaneum.

Resultados: Se reconoce aquí que una universidad internacional é organizada para satisfacer las ambiciones de dirigir estas instituciones. Paul Otlet y Henri La Fontaine, padres detrás de ese ideal, engendran estas instituciones inflar la cooperación intelectual internacional como un salto en particular. De hecho, se están sentando las bases de la normalización de las bibliotecas y ampliar su ámbito de aplicación a las asociaciones privadas. 
Conclusiones: Gracias a este diálogo permanente y la cooperación a gran escala, el universalismo de conocimiento alcanza un grado de logro sin precedentes, sin que, por lo tanto, imponer la paz.

Palabra clave: Instituto Internacional de Bibliografía. Mundaneum. Repertorio Bibliográfico Universal.

Recebido em: Abril de 2015

Aceito em: Julho de 2015 